\title{
Simulation of vortex shedding flows using high-order fractional step methods
}

\author{
M. P. Kirkpatrick* S. W. Armfield* J. H. Kent* \\ T. $\operatorname{Dixon}^{\dagger}$
}

(Received 7 August 2000)

\begin{abstract}
Unsteady flow past a square cylinder is simulated using a fractional step method to advance the Navier-Stokes equations in time. The fractional step method is a single step method whereby the momentum equations are solved using an explicit/implicit scheme and an
\end{abstract}

* Department of Mechanical and Mechatronic Engineering, The University of Sydney, Sydney, NSW 2006, Australia. mailto: elf@mech.eng.usyd.edu.au

$\dagger$ Sugar Research Institute, Mackay, Queensland 4741, Australia.

${ }^{0}$ See http://anziamj . austms.org. au/V42/CTAC99/Kirk for this article and ancillary services, (C) Austral. Mathematical Soc. 2000. Published 27 Nov 2000. 
approximate pressure field to yield an estimate of the velocity. This velocity is then projected onto a divergence free field using a pressure correction obtained by the solution of a Poisson pressure correction equation. The integration then proceeds to the next time step.

Results were obtained using a Crank-Nicolson scheme and hybrid second and third order Adams-Bashforth/Adams-Moulton schemes and second order in time behaviour is verified for velocities for a developed flow over a square cylinder. Results will be presented comparing the accuracy and efficiency of these schemes with unsteady flows of this type, as well as detailing some of the pitfalls that can be encountered with this approach.

\section{Contents}

1 Introduction

C858

2 Method

C859

2.1 Spatial Discretisation . . . . . . . . . . . . C860

2.2 Time Advancement of Momentum Equations . . . . . . . . C C860

2.2.1 Crank-Nicolson Scheme . . . . . . . . . . . C861

2.2.2 Hybrid Adams Schemes . . . . . . . . . . . C C862

2.2.3 Boundary conditions ............ . . C863

2.2.4 Pressure correction . . . . . . . . . . . . C865

3 Results

C866 
3.1 Driven Cavity . . . . . . . . . . . . . . . . C867

3.1.1 Case 1: Integration without pressure correction . . C867

3.1.2 Case 2: Integration with pressure correction . . . . C868

3.2 Square Cylinder . . . . . . . . . . . . . . C871

4 Conclusions

C873

References

C875

\section{Introduction}

The flow of an isothermal fluid is governed by equations for conservation of mass and conservation of momentum (the Navier-Stokes equations). When these equations are solved for an incompressible fluid, pressure provides coupling between the momentum and mass conservation equations. This coupled system can be solved iteratively using methods such as the SIMPLE procedure (Patankar [9]) or by a non-iterative method such as the fractional step method.

The fractional step method was first suggested by Harlow and Welch [3] and Chorin [4]. The scheme became popular after Kim and Moin [5] modified it for finite volumes on a staggered grid. Since then a number of versions of the scheme have been suggested. Of these, the pressure-correction method (Van Kan [6] and Bell and Colella [1]) was found to be the fastest of the 
methods tested by Armfield and Street [10] and is the method used here. Gresho [8] has shown analytically that this method is second order in time.

In this paper, the behaviour of a number of second and third order schemes for the time integration of the Navier-Stokes equations using the fractional step method has been investigated. The order of accuracy of the schemes for the momentum equations is demonstrated by integration of these equations alone for a driven cavity flow. The addition of a pressure correction step necessary to ensure a divergence free velocity field is shown to limit the accuracy of the overall method to second order.

Results have also been obtained for fully developed vortex-shedding flow over a square cylinder showing that second order accuracy is retained with all schemes for this case. Finally, the accuracy, stability and efficiency of the three schemes are compared. All results presented were obtained using the PUFFIN code (Particles IN Unsteady Fluid Flow).

\section{Method}

The Navier-Stokes equations for an incompressible fluid are written in the following form:

$$
\begin{aligned}
v_{t}+(v \cdot \nabla) v & =-\nabla P+\frac{1}{R e} \nabla^{2} v \\
\nabla \cdot v & =0
\end{aligned}
$$


where $v$ is the velocity, $P$ the pressure and Re the Reynolds number.

\subsection{Spatial Discretisation}

These equations are discretised in space using a finite volume formulation on a non-uniform, staggered, Cartesian grid. Derivatives are calculated using a second-order accurate central difference scheme.

An advantage of the central difference scheme over non-centred schemes is that it is relatively free of numerical dissipation. While this improves the accuracy of the scheme, it can also lead to non-physical oscillations if a grid of insufficient refinement is used. Patankar [9] found that the central difference scheme will give realistic solutions as long as the cell Peclet number, $P e=\frac{v \Delta h}{\Gamma}$, is kept less than two (where $\Delta h$ is the grid cell dimension and $\Gamma$ is a kinematic diffusion coefficient).

From the point of view of testing the time accuracy of a scheme, we found that it is possible to obtain good results with a $P e>2$, as long as the oscillations were significantly smaller than other structures in the flow.

\subsection{Time Advancement of Momentum Equations}

In the pressure-correction method, the momentum equations are first solved using the pressure gradient from the previous time step to give an approx- 
imate solution, $v^{*}$. We have developed a number of schemes for the integration of the momentum equations in time. These include: an iterative Crank-Nicolson scheme and non-iterative, second and third order hybrid explicit/implicit schemes. In the hybrid schemes, Adams-Bashforth methods are used for the advective terms and Adams-Moulton methods for the diffusive terms. These schemes will be referred to as CN, AB2/AM2 and AB3/AM3 respectively.

\subsubsection{Crank-Nicolson Scheme}

The CN scheme can be written as:

$$
\frac{v^{n+1}-v^{n}}{\Delta t}+\left[\frac{1}{2} H\left(v^{n+1}\right)+\frac{1}{2} H\left(v^{n}\right)\right]=-G p^{n}+\frac{1}{R e}\left[\frac{1}{2} L\left(v^{n+1}\right)+\frac{1}{2} L\left(v^{n}\right)\right]
$$

where $H$ is the discrete advection operator, $G$ the discrete gradient and $L$ the discrete Laplacian or diffusion operator.

When viscosity is constant, the diffusion operator is not dependent on time. As a result, solution of a pure diffusion equation such as the heat equation using the $\mathrm{CN}$ scheme does not require iteration to be second-order accurate. The non-linearity of the advection operator, however, makes it necessary to iterate through the momentum equations if second order accuracy is to be achieved. 


\subsubsection{Hybrid Adams Schemes}

This requirement of iterating can be avoided by using a hybrid explicit/implicit scheme. Advection terms are treated explicitly using an Adams-Bashforth method while diffusion terms are treated implicitly using an Adams-Moulton method. The second and third order hybrid schemes, AB2/AM2 and also AB3/AM3, are written as follows:

$$
\begin{aligned}
\frac{v^{n+1}-v^{n}}{\Delta t}+ & {\left[\frac{3}{2} H\left(v^{n}\right)-\frac{1}{2} H\left(v^{n-1}\right)\right]=-G p^{n}+\frac{1}{R e}\left[\frac{1}{2} L\left(v^{n+1}\right)+\frac{1}{2} L\left(v^{n}\right)\right] } \\
& \frac{v^{n+1}-v^{n}}{\Delta t}+\left[\frac{23}{12} H\left(v^{n}\right)-\frac{16}{12} H\left(v^{n-1}\right)+\frac{5}{12} H\left(v^{n-2}\right)\right] \\
= & -G p^{n}+\frac{1}{R e}\left[\frac{5}{12} L\left(v^{n+1}\right)+\frac{8}{12} L\left(v^{n}\right)-\frac{1}{12} L\left(v^{n-1}\right)\right]
\end{aligned}
$$

Because the non-linear terms are calculated at previous time steps (where all the necessary information is known) no iteration is required to achieve the "advertised" accuracy of the scheme.

The resulting matrix equation is solved using an ADI solver with the Thomas algorithm used to solve the tridiagonal systems.

A fully explicit scheme for both advection and diffusion terms has the advantage that no matrix inversion is required, however, the stability crite- 
rion for explicit treatment of diffusion terms is considerably more stringent than that for the advection terms. For diffusion terms the maximum useable time step is proportional to the characteristic diffusion time, $\frac{(\Delta h)^{2}}{\Gamma}$ (where $\Delta h$ is the minimum grid cell dimension and $\Gamma$ is a kinematic diffusion coefficient). For advection terms the maximum time step is proportional to the characteristic convection time, $\frac{\Delta h}{v}$. This condition is usually described in terms of the Courant number, $C=\frac{v \Delta t}{\Delta h}$. The limitation on the time-step due to the diffusion terms makes a fully explicit scheme impractical for most applications.

A disadvantage of Adams methods is that they require special treatment for the initial steps where no information about previous time-steps is available. We found that starting with a lower order method did not reduce the long-term accuracy of the scheme. The best results, however, were obtained by using $\mathrm{CN}$ for the first step.

An alternative approach which avoids this problem is to use a scheme such as Runge-Kutta in which each time step is divided into a series of substeps. This advantage comes at the expense of repeated computation of all the terms at each substep.

\subsubsection{Boundary conditions}

The boundary conditions for the driven cavity flow are solid boundaries with a no-slip condition. The velocity of the solid boundary may be zero or non- 
zero. Rather than using a phantom node, the tangential velocity node is placed on the solid boundary surface. As a result, the boundary velocity is constant and always exact.

In addition, the external flow past a square cylinder also requires inlet, outlet and periodic boundary conditions. The inlet boundary condition is applied by setting the normal velocity on the boundary to a constant value and using phantom nodes for the tangential velocity components. At the outlet boundary a convective outlet boundary condition is used,

$$
\frac{\partial v}{\partial t}+V_{b} \frac{\partial v}{\partial n}=0
$$

where $V_{b}$ is the bulk velocity. This boundary condition allows convection of structures out of the domain and avoids problems with reflection of pressure waves (Pauley et al. [7]).

At periodic boundaries all terms are treated explicitly. This keeps the matrices for each line tridiagonal. A modification of the Thomas algorithm for a tridiagonal matrix with periodic elements is approximately twice as expensive to solve as the standard form. For the present case, in which the cells close to the periodic boundaries are approximately an order of magnitude larger (in the boundary normal direction) than the smallest cells and velocity gradients at the boundary are small, explicit treatment of diffusion flux at the boundary was found not to cause stability problems. 


\subsubsection{Pressure correction}

After the approximate solution for the velocity field, $v^{*}$, has been obtained by integration of the momentum equations using one of the schemes above, mass conservation is enforced through a pressure correction step. The approximate velocity field is projected onto a subspace of divergence free velocity fields. The projection is achieved by solving a Poisson equation for the pressure correction, $\phi$, in which the source term is the divergence of velocity in each cell:

$$
L \phi=\frac{1}{\Delta t} D v^{*}
$$

The pressure correction is used to correct the velocity field,

$$
v^{n+1}=v^{*}-\Delta t G \phi
$$

and the pressure field,

$$
p^{n+1}=p^{n}+\phi
$$

It has been shown (Shah [2]) that the Poisson equation for the pressurecorrection method is independent of boundary values of $v^{*}$ and $\phi$. As a result, the use of intermediate values of velocity, $v^{*}$, at the boundaries does not affect the order of accuracy of the overall scheme as it did with earlier fractional step methods. 
It also enables us to set special boundary conditions on the Poisson equation to drive the net mass flux across a boundary to a desired (zero or nonzero) value. Reverting to dimensional form, for a desired net mass flux across the boundary, $\dot{m}_{b}$, the following boundary condition is used,

$$
\frac{\delta \phi}{\delta n}=\frac{-\left(\dot{m}_{b}-\sum\left(\varrho v^{*} \delta A\right)\right)}{\delta t A_{b}}
$$

where $\delta A$ is the boundary normal area of each cell and $A_{b}$ is the total area of the boundary.

This boundary condition sets the normal gradient of the pressure correction across the boundary to a value which gives the velocity correction required to achieve the correct net mass flux. This boundary condition was developed to enable us to apply periodic boundary conditions with a non-zero net mass-flux. It also accelerates the solution of the Poisson equation.

The Poisson equation is solved using an ADI solver. The divergence of the corrected velocity field is checked and the correction procedure repeated until the required tolerance is achieved.

\section{Results}

Results have been obtained for the three schemes, CN, AB2/AM2 \& AB3/AM3 using the following test cases: 
- start-up flow in a driven-cavity without the pressure correction step;

- start-up flow in a driven-cavity with the pressure correction step;

- developed flow over a square cylinder.

Errors were evaluated using an $\mathrm{L}_{2}$ norm of the difference between the test solution and a benchmark solution obtained with a Courant number four times less than the smallest Courant number used for the tests.

\subsection{Driven Cavity}

Start-up flow in a driven cavity was integrated at a Reynolds number of 400 based on lid velocity and cavity height. Boundary conditions were stationary solid boundaries at the base and sides of the cavity and a solid boundary with non-zero tangential velocity for the lid. A $32 \times 32$ uniform grid was used. Integration times, $t$, are non-dimensionalised by the lid velocity and the cavity height.

\subsubsection{Case 1: Integration without pressure correction}

The momentum equations alone were integrated over $t=0$ to 0.05 . The benchmark run was performed with a time step corresponding to a Courant 
number $C=0.00125$. Tests were performed with time steps ranging from $C=$ 0.005 to 0.04 . For this test, one of the side walls was also given a tangential velocity in order to obtain a non-zero field for both velocity components.

Figure 1 shows errors of the three schemes for the horizontal velocity, $u$. (Results for $v$ were identical.) These results show that the solution of the momentum equations by themselves is second order accurate with the $\mathrm{CN}$ and AB2/AM2 schemes and third order accurate with the AB3/AM3 scheme.

\subsubsection{Case 2: Integration with pressure correction}

The momentum equations were integrated and the pressure correction applied over $t=0$ to 0.5 . The benchmark run was performed with a time step corresponding to a Courant number $C=0.0125$. Tests were performed with time steps ranging from $C=0.05$ to 0.4 .

Figure 2 shows errors of the three schemes for two velocity components, $u$ and $v$. With the pressure correction applied, the second order schemes remain second order but the accuracy of AB3/AM3 is reduced from third to second order. This agrees with the theoretical findings of Gresho [8] that the pressure correction method is, at best, second order. 


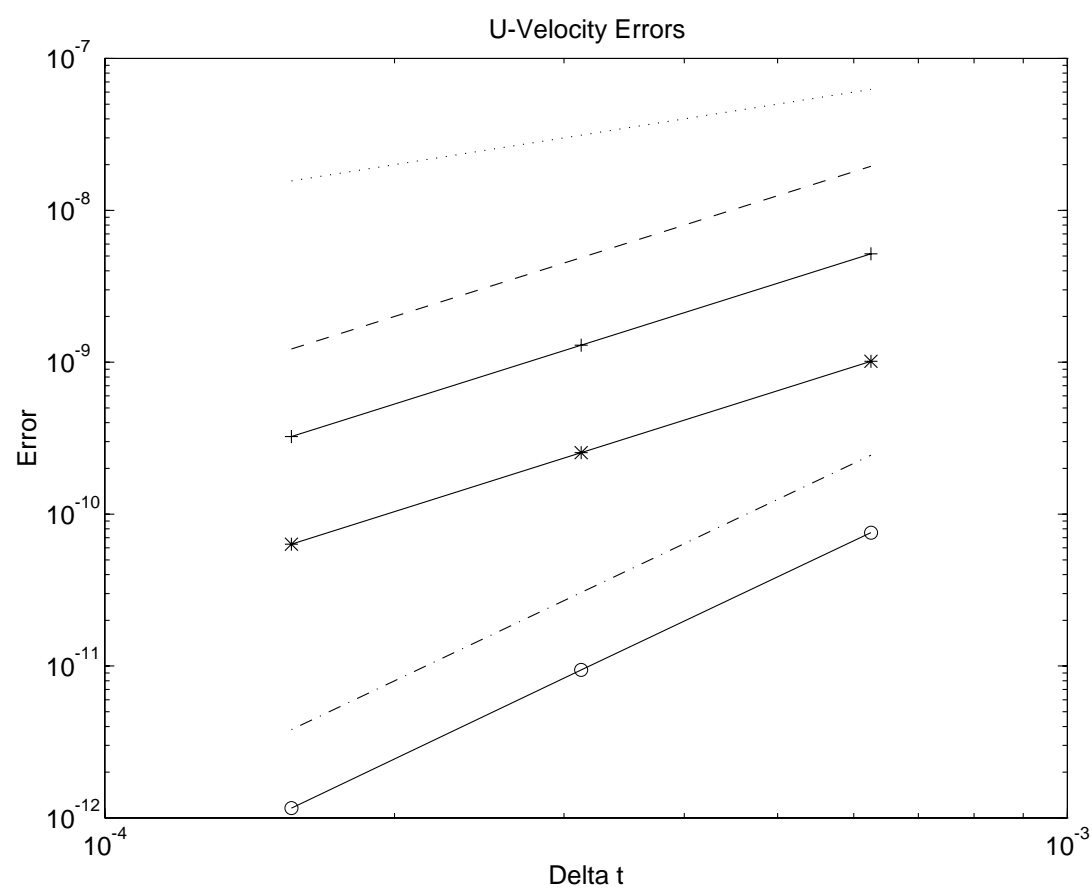

FIGURE 1: Velocity errors for driven cavity - no pressure correction: $[+\mathrm{CN}$, * AB2/ АM2, о АB3/ Ам3, lines ... 1st order, _ _ 2nd order, _. _ 3rd order] 

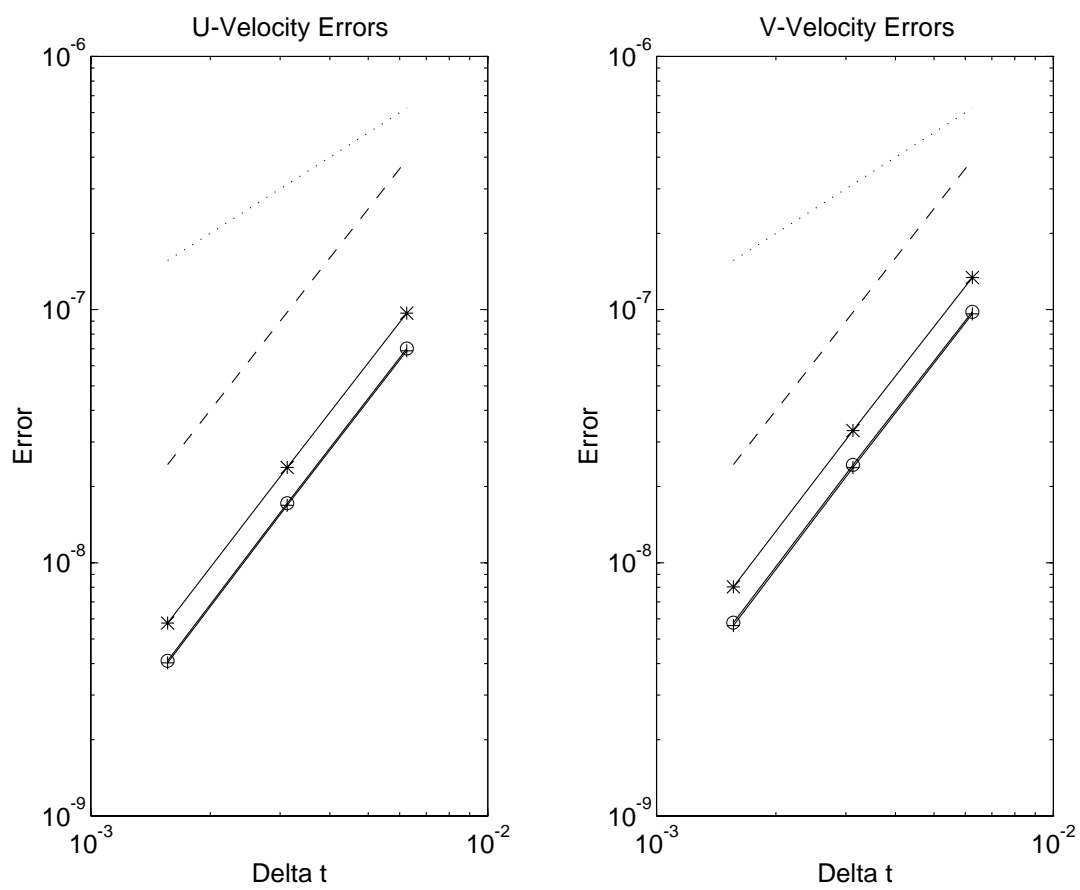

FIGURE 2: Velocity errors for driven cavity - with pressure correction: [+ $\mathrm{CN},{ }^{*} \mathrm{AB} 2 / \mathrm{AM} 2$, о AB3/AM3, lines ... 1st order, _ _ 2nd order] 


\subsection{Square Cylinder}

Flow over a square cylinder at a Reynolds number of 50 was integrated on a non-uniform $72 \times 216$ grid. The cylinder of width, 1, was placed in a domain of spatial size $9 \times 17$. The grid was finest close to the cylinder with a resolution of 24 cells along each face. From here the grid was expanded in both directions using a geometric series. The grid was designed with the aim of keeping the cell Peclet number $P e \sim 2$ throughout as much of the domain as possible. The maximum value is $P e \sim 8$ at the outlet.

In the streamwise direction, inlet and a convective outlet boundaries were used while periodic boundaries were used in the cross-stream direction. Integration times, $t$, are non-dimensionalised by the bulk velocity and the cylinder width.

The initial conditions were velocity and pressure fields for developed flow. These fields were obtained by integrating the flow from an initial divergence free velocity field at $t=0$ to developed flow at $t=40$. A plot of iso-vorticity lines for this field is shown in Figure 3. The effect of the higher Peclet number close to the outlet is seen in the small wiggles in the solution in this area. This did not affect the time accuracy results.

Figure 4 shows errors of the three schemes for two velocity components, $u$ and $v$. They show all the schemes retain second order accuracy for a vortexshedding flow. The error of AB3/AM3 and CN is seen to be approximately half that of AB2/AM2 for $u$-velocity. Errors were similar with all schemes for 


\section{AIR}

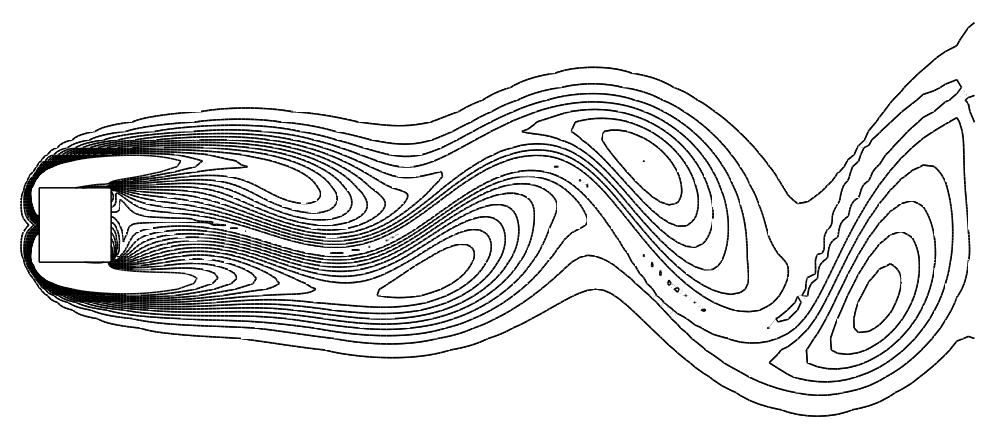

Figure 3: Flow over a square cylinder - vorticity 
$v$-velocity.

The stability limit for all schemes was found experimentally to be $C \sim 1.0$.

\section{Conclusions}

Three high order schemes for time-integration of the unsteady Navier-Stokes equations using a fractional step method have been investigated. It has been demonstrated that the third order hybrid scheme, is reduced to second order when used in combination with the fractional step method. This is because the fractional step method is first order accurate for pressure.

Second order accuracy has been obtained for developed vortex-shedding flow past a square cylinder with all schemes. AB3/AM3 has been demonstrated to be the most accurate scheme for this flow, though still second order.

The hybrid schemes are of similar accuracy to the Crank-Nicolson scheme and more than twice as fast in the momentum equation integration step. The errors of the third order hybrid scheme are similar to the second order scheme for this flow. The stability limit for all schemes was $C \sim 1.0$.

The additional computational costs of the third order scheme are very small, however the increase in storage requirements are considerable. 

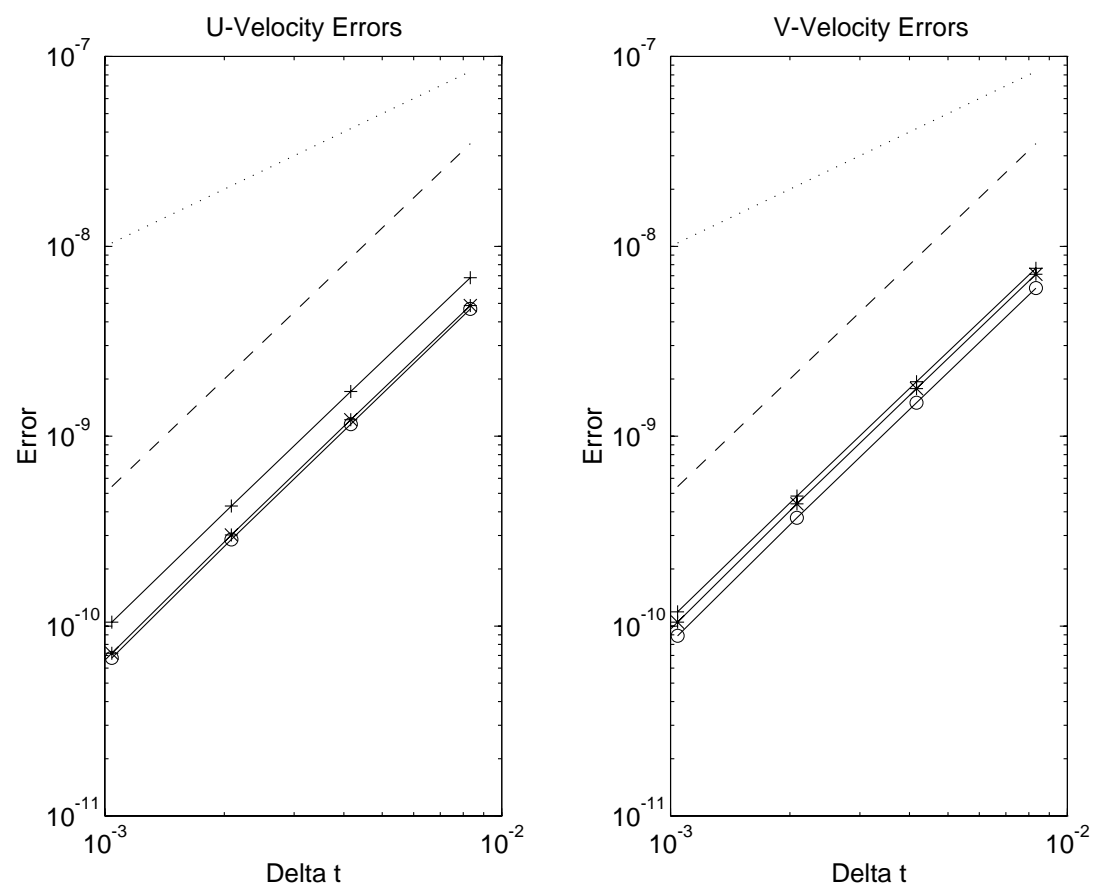

FIGURE 4: Velocity errors for square cylinder - with pressure correction: [+ $\mathrm{CN},{ }^{*} \mathrm{AB} 2 / \mathrm{AM} 2$, o AB3/AM3, lines ... 1st order, _ _ 2nd order] 
We conclude that the second order hybrid scheme offers the best performance for problems of this type.

\section{References}

[1] J. B. Bell and P. Colella. A second-order projection method for the incompressible Navier-Stokes equations. J. Comp. Physics, 85:257-283, 1989. C858

[2] K. B. Shah. Large Eddy Simulations of Flow Past a Cubic Obstacle. PhD thesis, Stanford University, 1998. C865

[3] F. Harlow and E. Welch. Numerical calculation of time-dependent viscous incompressible flow of fluid with free surface. Physics of Fluids, 8:2182-2189, 1965. C858

[4] A. J. Chorin. Numerical solution of the Navier-Stokes equations. Math. Comput., 22:745-762, 1968. C858

[5] J. Kim and P. Moin. Application of a fractional step method to incompressible Navier-Stokes equations. J. Comp. Physics, 59:308-323, 1985. C858

[6] J. Van Kan. A second order accurate pressure correction scheme for viscous incompressible flow. SIAM J. Sci. Stat. Comput., 7:870-891, 1986. C858 
[7] L. L. Pauley, P. Moin, and W. C. Reynolds. The structure of two-dimensional separation. J. Fluid Mech., 220:397-411, 1990. C864

[8] P. Gresho. On the theory of semi-implicit projection methods for viscous incompressible flow and its implementation via finite element method that also introduces a nearly consistent mass matrix. part 1 : Theory. Int. J. Numerical Methods in Fluids, pages 587-620, 1990. C859, C868

[9] S. V. Patankar. Numerical Heat Transfer and Fluid Flow. Taylor and Francis, 1980. C858, C860

[10] S. W. Armfield and R. Street. The fractional-step method for the Navier-Stokes equations on staggered grids: The accuracy of three variations. J. Comp. Physics, submitted, 1999. C859 